\title{
Fitting mixed models to messy longitudinal data: A case study involving estimation of post mortem intervals
}

\author{
Julio M. Singer ${ }^{\mathrm{a}}$, Francisco M. M. Rocha ${ }^{\mathrm{b}}$, \\ Carmen D. S. Andréa and Talita Zerbini ${ }^{\mathrm{a}}$ \\ ${ }^{\mathrm{a}}$ Universidade de São Paulo \\ ${ }^{\mathrm{b}}$ Universidade Federal de São Paulo
}

\begin{abstract}
Non-linear mixed models are useful in many practical longitudinal data problems, especially when they are derived as solutions to differential equations generated by subject matter theoretical considerations. When this underlying rationale is not available, practitioners are faced with the dilemma of choosing a model from the numerous ones available in the literature. The situation is even worse for messy data where interpretation and computational problems are frequent. This is the case with a pilot observational study conducted at the School of Medicine of the University of São Paulo in which a new method to estimate the time since death (post-mortem interval-PMI) is proposed. In particular, the attenuation of the density of intra-cardiac hypostasis (concentration of red cells in the vascular system by gravity) obtained from a series of tomographic images was observed in the thoraces of 21 bodies of hospitalized patients with known time of death. The images were obtained at different instants and not always at the same conditions for each body, generating a set of messy data. In this context, we consider three ad hoc models to analyse the data, commenting on the advantages and caveats of each approach.
\end{abstract}

\section{Introduction}

The estimated time since death, known as post-mortem interval (PMI), is fundamental in many instances such as in criminal investigations, because its determination may be used to acquit or condemn a suspect, as the data is compared with the alibis provided by those under investigation. Standard estimates of PMI based on the evaluation of some physical characteristics have margins of error of 2 hours during the first 6 hours following death, of 3 hours in the next 14 hours and of 4.5 hours in the subsequent 10 hours as indicated by Kaliszan et al. (2009). These authors mention that many alternatives to improve the determination of the PMI have been attempted but they have not been regularly used in practice.

The concentration of red cells in the vascular system and the diffusion of plasma into the adjacent tissues after death result in a reddish-purple color, a phenomenon

Key words and phrases. Autopsy, calibration, computed tomography, diagnostics, hypostasis, linear mixed models, post-mortem interval, residual analysis.

Received September 2016; accepted October 2017. 
called hypostasis [see Knight (1991), Sannohe (2002) or Dolinak et al. (2005), for example] or livor mortis [Thali (2003)]. According to Dolinak et al. (2005), hypostasis begins to develop at the moment of cardiac arrest, becoming perceptible after 3-4 hours and more obvious after 6-8 hours, with full development in the skin occurring after 10-12 hours. Fávero (1991), on the other hand, suggests that the first signs of hypostasis occur within 10 minutes after death, becoming obvious after 1 to 3 hours and stabilizing after approximately 8 to 12 hours. Such differences in tissue density (attenuation) may be visualized via post-mortem computer tomography (PMCT). Intra-cardiac hypostasis is caused by the gradual sedimentation of the cellular elements of the blood in the heart and in the great vessels, allowing visualization in the superior vena cava, the right atrium, the right ventricle, the thoracic aorta, the left atrium and the left ventricle. Ishida et al. (2011) examined 50 cases, concluding that intra-cardiac hypostasis was easily observed in $55 \%$ of the patients, with the best sites for analysis being the right atrium ( $88 \%)$, the left atrium $(88 \%)$ and the thoracic aorta $(76 \%)$.

To our knowledge, authors of studies involving PMCT virtual autopsy like Shiotani et al. (2002), Levy et al. (2010) or Ishida et al. (2011) did not consider using complementary imaging to facilitate PMI estimation; published studies concerning tomographic analysis of hypostasis in the heart and in the great vessels use a single image for each subject, rather than a series of timed images at increasing post-mortem instants. Our objective is to discuss statistical methodology to analyze data from a pilot study designed to estimate the PMI based on a series of tomographic analyses of intra-cardiac hypostasis.

In Section 2, we describe the pilot study identifying aspects that must be taken into account for analysis. Three alternative models are presented in Section 3 and the results of the data analyses are considered in Section 4. We conclude with a brief discussion in Section 5.

\section{The study}

We consider a prospective observational pilot study conducted at the School of Medicine of the University of São Paulo, Brazil and approved by its Ethics Committee. The data were collected from 21 corpses of patients of both sexes who died while under medical care and were routed to autopsy. This guarantees the knowledge of the exact time of death. For each subject, demographic data (age, sex and ethnicity), disease history, the hypothetical cause of death and the time of death observed by the physician were recorded. A pathologist was responsible for specifying the causes of death as well as writing the autopsy reports.

The bodies were placed in dorsal decubitus position with the arms placed behind the head to allow transition through the tomograph, to bypass cadaveric rigidity and to reduce the emission of radiation. Images were obtained at post-mortem 
intervals of between one and twenty hours, with the examination repeated at intervals of one hour, depending on the availability of the tomographic equipment. The images were analyzed simultaneously by a forensic physician and by a radiologist and were reviewed by a pulmonary pathologist. The acquired images were analyzed one by one, with the goal of choosing the most appropriate slice for the attenuation measurements. Each cardiac chamber was divided into two zones (anterior and posterior) and measurements were obtained in approximately spherical portions with average volume of $1.4 \mathrm{~cm}^{3}$ within each zone. At each observation instant, the average tissue density (in Hounsfield units) of each segment was computed and the attenuation values in each area were determined.

The difference between the attenuation measurements obtained from the Anterior segment of the Right atrium and the Posterior segment of the Left atrium [labeled dif(ARPL)] in each selected image was used for statistical analysis. This difference corresponds to the greatest distance between the measured points within the heart and has been considered in previous studies [see Ishida et al. (2011), for example]. Given that hypostasis begins when the heart stops beating, the densities of the anterior and posterior zones of the atrium were considered equal at the moment of death.

Anthropometric data for the 21 subjects as well as the initial and final PMI at which the images were obtained and are summarized in Table 1.

The subjects included 12 males and 9 females, with an average age of 55.5 years (minimum of 19 and maximum of 92). The first measurement in each body ranged from one to twenty hours post-mortem and different numbers of images were obtained in each subject because of the demand for the autopsy room. The temporal evolution of the observed measurements of dif(ARPL) is displayed in Figure 1.

\section{The models}

Given that hypostasis tends to stabilize after a certain period, it seems reasonable to adopt non-linear models with an asymptote to represent the data. Differently from many cases addressed in the literature [see Davidian and Giltinan (2003), for example], there is no known mechanistic model to describe the attenuation process. We are then faced with the problem of choosing an ad hoc model that can take the characteristics of the phenomenon under investigation into account. This is a difficult task in view of the numerous alternatives available in the literature.

A special case of the Mitscherlich model (known in the literature as von Bertalanffy growth model) and multiplicative exponential models were considered as candidates to represent the relation between the response [dif(ARPL)] and time (PMI). Furthermore, the different behaviour observed for the different subjects (see Figure 1) justifies the use of mixed models. For details on these models, the 
Table 1 Epidemiological data and the initial (PMIi) and final (PMIf) PMI at which the images were obtained

\begin{tabular}{lcccccc}
\hline Subject & Sex & Race & $\begin{array}{c}\text { Age } \\
\text { (years) }\end{array}$ & $\begin{array}{c}\text { PMIi } \\
\text { (hours) }\end{array}$ & $\begin{array}{c}\text { PMIf } \\
\text { (hours) }\end{array}$ & $\begin{array}{c}\text { Number of } \\
\text { observations }\end{array}$ \\
\hline 1 & Male & Caucasian & 26 & 4 & 4 & 1 \\
2 & Male & Black & 82 & 3 & 3 & 1 \\
3 & Female & Black & 53 & 4 & 7 & 4 \\
4 & Male & Caucasian & 44 & 13 & 14 & 2 \\
5 & Male & Caucasian & 37 & 7 & 7 & 1 \\
6 & Female & Caucasian & 67 & 12 & 13 & 2 \\
7 & Male & Black & 59 & 10 & 12 & 3 \\
8 & Female & Black & 83 & 19 & 20 & 2 \\
9 & Female & Mixed & 48 & 20 & 20 & 1 \\
10 & Female & Caucasian & 84 & 10 & 11 & 2 \\
11 & Male & Mixed & 68 & 8 & 9 & 2 \\
12 & Male & Mixed & 31 & 11 & 13 & 3 \\
13 & Female & Caucasian & 92 & 1 & 4 & 4 \\
14 & Male & Caucasian & 79 & 4 & 6 & 3 \\
15 & Male & Caucasian & 21 & 3 & 10 & 3 \\
16 & Female & Mixed & 68 & 12 & 14 & 3 \\
17 & Female & Caucasian & 49 & 12 & 14 & 9 \\
18 & Female & Caucasian & 19 & 8 & 16 & 4 \\
19 & Male & Caucasian & 47 & 8 & 11 & 3 \\
20 & Male & Caucasian & 50 & 11 & 13 & 3 \\
21 & Male & Mixed & 53 & 11 & 13 & \\
\hline
\end{tabular}

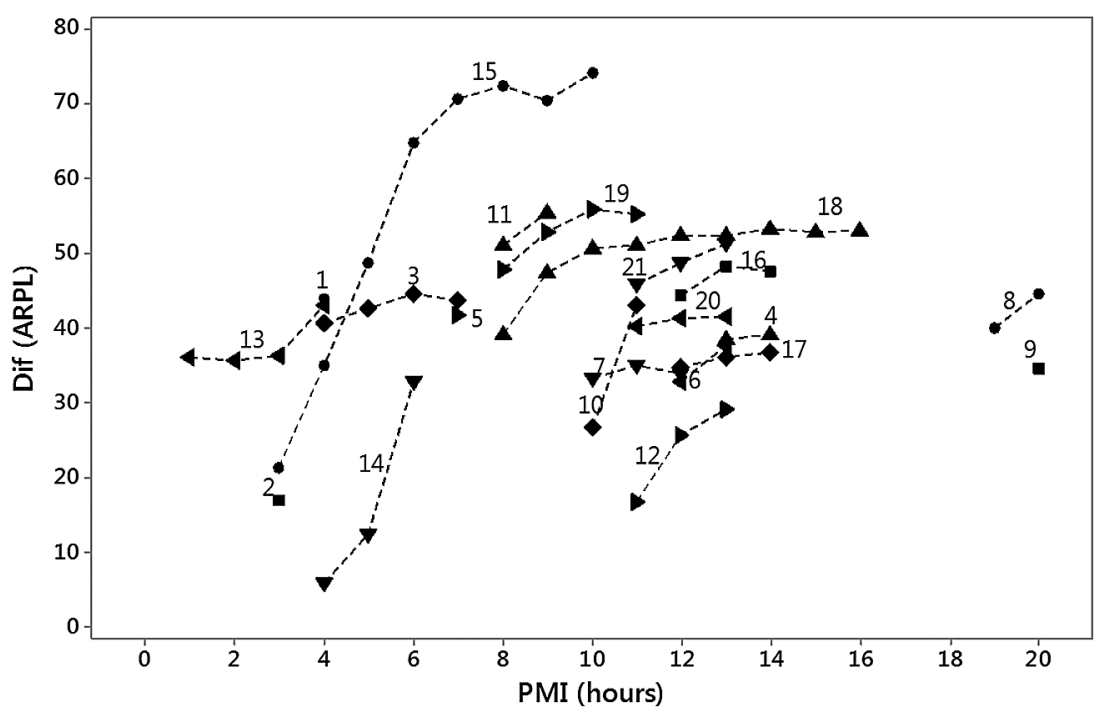

Figure 1 Individual profiles of the attenuation difference between the anterior segment of the right atrium and the posterior segment of the left atrium [dif(ARPL)]. 
reader is referred to Lindsey (2004), Fitzmaurice et al. (2011) or to Demidenko (2013), among others.

The von Bertalanffy mixed model may be expressed as

$$
y_{i j}=\left(\gamma+g_{i}\right)\left\{1-\exp \left[-\left(\delta+d_{i}\right) x_{i j}\right]\right\}+u_{i j}, \quad x_{i j} \geq 0,
$$

where $y_{i j}$ denotes the $j$ th response of the $i$ th subject, $x_{i j}$ denotes the corresponding time where this response was observed, $\gamma>0$ and $\delta>0$ are parameters, the random effects $\mathbf{g}_{i}=\left(g_{i}, d_{i}\right)^{\top}$ follow independent Gaussian distributions with mean vector $\mathbf{0}$ and covariance matrix $\mathbf{G}$ and $f_{i j}$ are random errors following independent Gaussian distributions with mean 0 and variance $\sigma^{2}$. Furthermore, $\mathbf{g}_{i}$ and $u_{i j}$ are considered independent. Here, the parameter $\gamma$ represents the asymptote or the value at which the response stabilizes and $\delta$ is a parameter related to the rate with which the asymptote is reached (known as the intrinsic growth parameter). As discussed in Vonesh and Chinchilli (1996, Ch. 7), among others, the parameters $\gamma$ and $\delta$ in (3.1) have a subject-specific interpretation, that is, they represent the behaviour of the response for a "typical" subject (one for which the random effects are equal to zero) and not a population-averaged response. Although population-averaged response curves may be obtained by integrating out the random effects, estimates of the corresponding parameters are obtainable only in very special cases.

The multiplicative exponential mixed model is expressed as

$$
y_{i j}=\left(\alpha a_{i}\right) \exp \left[-\left(\beta+b_{i}\right) / x_{i j}\right] e_{i j}, \quad x_{i j}>0
$$

with parameters $\alpha$ and $\beta$ having similar interpretations as those in the von Bertalanffy mixed model but refer to population-averaged characteristics as opposed to subject-specific ones. Note that (3.2) may be linearized as

$$
y_{i j}^{*}=\alpha^{*}+a_{i}^{*}+\left(\beta^{*}+b_{i}\right) x_{i j}^{*}+e_{i j}^{*}, \quad x_{i j}^{*}>0,
$$

where $y_{i j}^{*}=\log \left(y_{i j}\right), \alpha^{*}=\log (\alpha), \beta^{*}=-\beta, a_{i}^{*}=\log \left(a_{i}\right), e_{i j}^{*}=\log \left(e_{i j}\right)$ and $x_{i j}^{*}=1 / x_{i j}$. Assuming that the random effects $\mathbf{b}_{i}^{*}=\left(a_{i}^{*}, b_{i}\right)^{\top}$ follow independent Gaussian distributions with mean vector $\mathbf{0}$ and covariance matrix $\mathbf{G}^{*}$ and $e_{i j}^{*}$ are random errors following independent Gaussian distributions with mean 0 and variance $\tau^{2}$, (3.3) may be classified as a standard linear mixed model. According to this specification, in (3.2), $e_{i j}$ follows a log-normal distribution and both $\alpha$ and $a_{i}$ are positive implying that the predicted responses are positive, a feature required by the problem under investigation.

Alternatively, the evolution of $\operatorname{dif}(\mathrm{ARPL})$ could be represented by a mixed segmented regression model with random change-points like

$$
y_{i j}=\kappa_{i}\left\{\psi_{i}+\left(x_{i j}-\psi_{i}\right) I_{\left\{x_{i j} \leq \psi_{i}\right\}}\right\}+e_{i j}
$$

where $\kappa_{i}=\kappa+k_{i}, \psi_{i}=\psi+p_{i}, e_{i j} \sim N\left(0, \sigma^{2}\right)$ and $\mathbf{b}_{i}=\left(k_{i}, p_{i}\right)^{\top} \sim N[\mathbf{0}, \mathbf{G}(\boldsymbol{\theta})]$ with $\mathbf{G}$ denoting an unstructured covariance matrix with parameters $\boldsymbol{\theta}=\left(\sigma_{k}^{2}, \sigma_{p}^{2}\right.$, $\left.\sigma_{k p}\right)^{\top}$ are independent random variables. Here, $\kappa$ denotes the slope ("rate") with 
which the response $\operatorname{dif}(\mathrm{ARPL})$ stabilizes, and $\psi$ indicates the time at which this occurs. The level at which the response stabilizes ("asymptote") corresponds to $\kappa_{i} \psi_{i}$ Given its non-linear nature, the parameters in this model have the same interpretational characteristics as those defining the von Bertalanffy model, that is, the parameters refer to the characteristics of a "typical" subject (corresponding to $\left.\mathbf{b}_{i}=\mathbf{0}\right)$.

Model (3.1) is intrinsically non-linear and is derived from differential equations with interesting interpretations as described in Lindsey (2004, p. 273). It may be fitted with standard methodology for non-linear mixed models as indicated in Pinheiro et al. (2014). Model (3.4) is attractive because of its simplicity but requires specialized algorithms. We motivate and describe an adaptation of an algorithm in Muggeo et al. (2014) in the Appendix. Model (3.2) may be linearized, yielding (3.3) and as such may be fitted via standard linear mixed models methodology outlined in Demidenko (2013), for example. Furthermore, diagnostic tools for linear mixed models are well disseminated in the literature.

Note that for biological reasons, the intercept of the three models are constrained to zero but this is not necessary in a more general setting.

\section{Results}

Initially, we considered restricted maximum likelihood methods (REML) to fit variations of the three models obtained by including or not random effects associated to each parameter using the package nlme [Pinheiro et al. (2014)] of the $R$ software [R Core Team (2014)] as well as the algorithm outlined in the Appendix for the segmented regression model. The results are displayed in Table 2.

Fitting the von Bertalanffy (3.1) or the segmented (3.4) regression models requires good initial estimates, a difficult task especially because the search involves two or three parameters; in some instances convergence is not attained and in other cases, different starting values lead to different solutions. In general, such problems do not occur when fitting the exponential model (3.2) in the linearized form (3.3). This last model has the advantage of allowing comprehensive diagnostic tools as indicated in Singer et al. (2017) who developed $\mathrm{R}$ functions for such purposes (available from www.ime.usp.br/ jmsinger/lmmdiagnostics.zip). Given the lack of theoretical reasons underlying the structure of the phenomenon under study, such tools may have a limited role regarding the choice of the most appropriate class of models, but may well serve to a fine tuning of the chosen one. Furthermore, the exponential model allows the estimation (prediction) of the random effects via the solution of Henderson's equations as opposed to genuinely non-linear models, that require iterative methods for such purposes. Details may be obtained in Lindstrom and Bates (1990), Vonesh and Chinchilli (1996, Ch. 7) and Singer et al. (2017), for example. Based on these considerations as well as on the descriptive analysis, that suggests different asymptotes and different rate parameters for different subjects 
Table 2 Parameter estimates, standard errors (Std error) and goodness of fit criteria

\begin{tabular}{lccccccc}
\hline Model & $\begin{array}{c}\text { Random } \\
\text { effects }\end{array}$ & $\begin{array}{c}\text { Estim } \\
\text { asympt }\end{array}$ & $\begin{array}{c}\text { Std } \\
\text { error }\end{array}$ & $\begin{array}{c}\text { Estim } \\
\text { rate }\end{array}$ & $\begin{array}{c}\text { Std } \\
\text { error }\end{array}$ & AIC & BIC \\
\hline von Bertalanffy & asymptote & 48.25 & 4.86 & 0.19 & $0.04^{\diamond}$ & 470.89 & 479.53 \\
von Bertalanffy & rate & 97.76 & 17.85 & 0.06 & $0.02^{\diamond}$ & 478.94 & 487.45 \\
von Bertalanffy & both & 47.33 & 3.81 & 0.25 & $0.05^{\diamond}$ & 479.19 & 491.96 \\
Exponential & asymptote & $42.79^{\star}$ & $3.96^{\diamond}$ & 0.92 & 0.36 & 52.23 & 60.74 \\
Exponential & rate & $64.64^{\star}$ & $6.21^{\diamond}$ & 4.78 & 1.11 & 34.09 & 42.60 \\
Exponential & both & $60.91^{\star}$ & $11.03^{\diamond}$ & 3.55 & 1.46 & -0.97 & 11.80 \\
Segmented & rate & $47.85^{\bullet}$ & 4.06 & 5.90 & 0.52 & 486.55 & 495.06 \\
Segmented & rate \& & & & & & & \\
& change-point & $52.79^{\nabla}$ & 3.77 & 5.31 & 0.69 & 496.81 & 509.57 \\
\hline
\end{tabular}

$\star$ : computed as $\exp \left(\alpha^{*}\right)$

$\diamond:$ computed via the Delta method

$\bullet:$ computed as rate $\times$ change-point $(=5.90 \times 8.10)$

$\nabla:$ computed as rate $\times$ change-point $(=5.31 \times 9.95)$

(see Figure 1), the chosen models for subsequent analyses included random effects associated with both parameters.

We considered the diagnostic plots described in Singer et al. (2017) to evaluate the fit of the linearized exponential mixed model. A plot of the modified LesaffreVerbeke index for the chosen model is displayed in Figure 2, suggesting that the adopted covariance structure is not appropriate for subjects labeled 10, 12 and 14. Also, a plot of the elements of the generalized random component leverage matrix (Figure 3) suggests that subject 13 is influential with respect to the estimates of the elements of the covariance matrix. A reasonable strategy for analysis is to modify the model to accommodate these differences in the covariance structure given that other diagnostic tools depend on good estimates of the associated covariance matrices.

These plots suggest that possibly a completely heteroskedastic model should be considered. However, the over-parametrization of such a model along with the small number of observations for many subjects lead to convergence problems. An alternative is to seek for more parsimonious models trying to identify for which subjects a different variance component should be included. This may be accomplished by iteratively fitting models with different variances for the subjects identified via diagnostic procedures.

With this in mind, the model was modified to accommodate different variances for the error term associated with the measurements of subjects 10, 12, 13 and 14 and refitted to the data. In the next step, subject 13 was still identified as influential with respect to the estimates of the elements of the covariance matrix (see Figure 4) although with less intensity as in the previous model and subject 15 showed up as a candidate for a different covariance component (see Figure 5). 


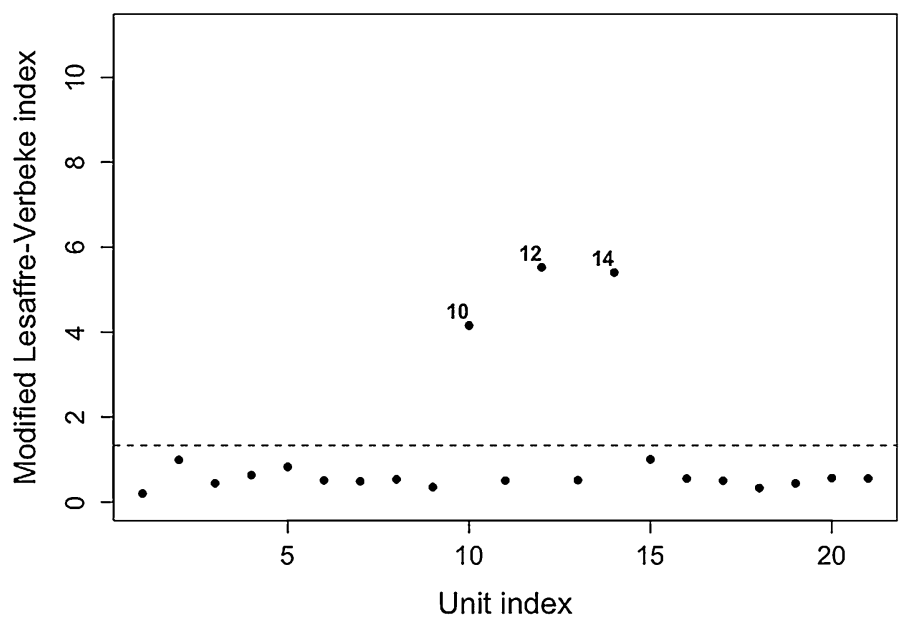

Figure 2 Plot of the modified Lesaffre-Verbeke index (initial model). Dashed line: 3 rd quartile + 1.5 interquartile range.

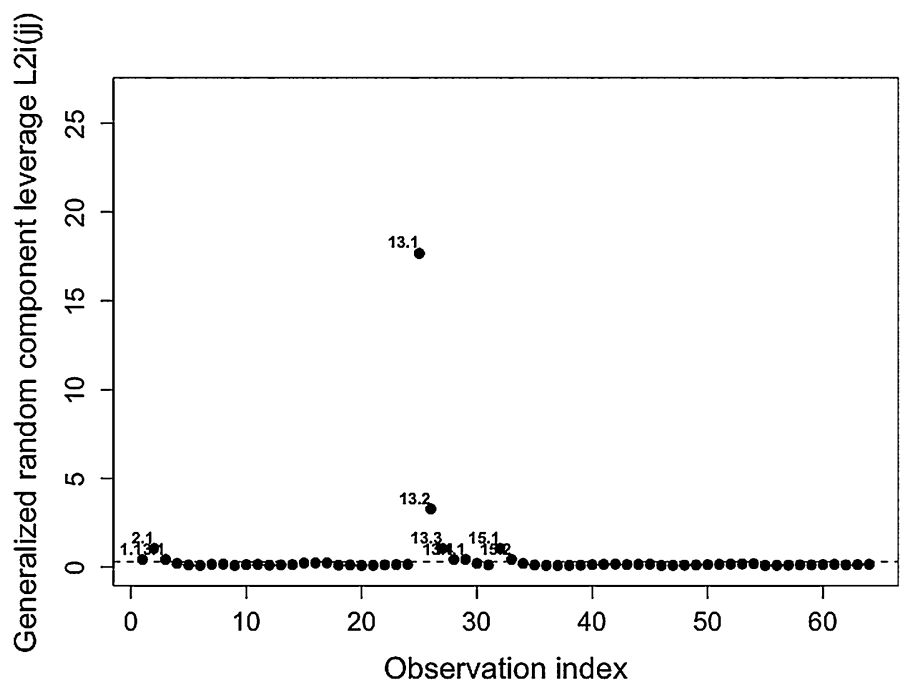

Figure 3 Plot of the generalized random component leverage (initial model). Dashed line: $3 \mathrm{rd}$ quartile +1.5 interquartile range.

This process was repeated once more, since residual analysis for the modified model still showed that for subjects 13 and 15 the covariance structure may not be appropriate. Note that the profile for subject 13 exhibits a convex rather than the expected concave shape (see Figure 1). Also, the first observation of subject 15 (which is the one with the second largest number of observations) is highlighted as the possible cause of the poor fit of the covariance structure. 


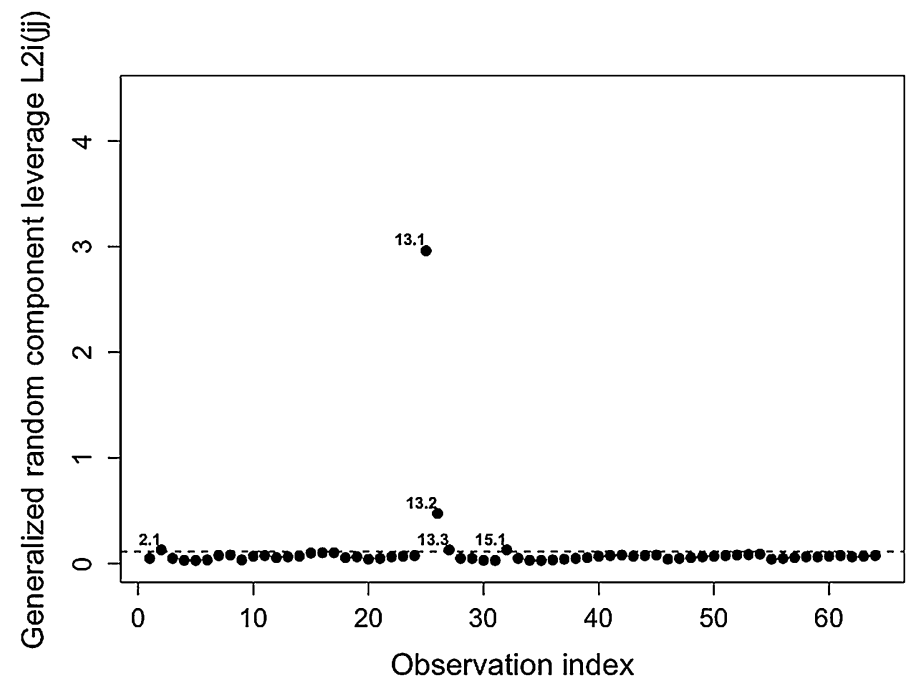

Figure 4 Plot of the generalized random component leverage (model with different covariance components for subjects 10, 12, 13 and 14). Dashed line: 3 rd quartile +1.5 interquartile range.

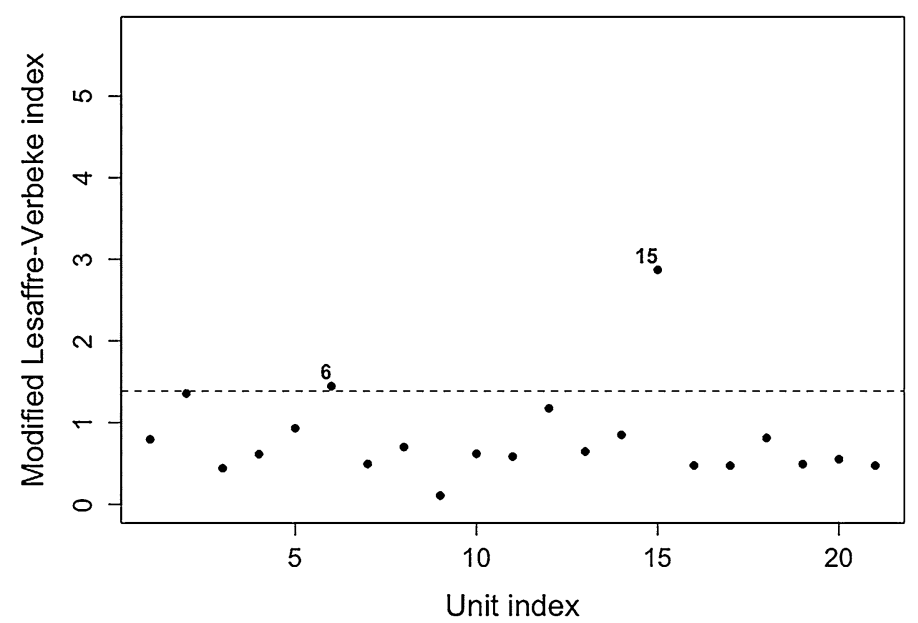

Figure 5 Plot of the modified Lesaffre-Verbeke measure (model with different covariance components for subjects 10,12, 13 and 14). Dashed line: 3 rd quartile +1.5 interquartile range.

Although one cannot expect an optimal model for data with the same nature as those under investigation, we specified a model that included different variance components for subjects 10,12,14 and 15 after excluding subject 13 and the first observation of subject 15. The residual plots for this model (see Figures A.1-A.5 in the Appendix) suggest that the assumption of normality is reasonable for both the random effects and the error terms, that the covariance structure is acceptable 
for all subjects and that there are no outliers. Subject 2 is highlighted as possibly influential, but one may not expect a perfect fit with this sort of sparse and irregular data, specially given that subject 2 has a single observation.

For this model, the (marginal) AIC $=-49.78$ and the $\mathrm{BIC}=-29.35$ (in contrast with $\mathrm{AIC}=-0.97$ and $\mathrm{BIC}=11.80$ for the original model) and the corresponding estimates ( \pm standard errors) for the population averaged asymptote $(\alpha)$ and speed related parameter $(\beta)$ are respectively $\widehat{\alpha}=52.7 \pm 5.6$ and $\widehat{\beta}=1.99 \pm 0.68$.

Because the objective of the proposed method is to estimate the PMI for a fixed value of $\operatorname{dif}(\mathrm{ARPL})$ for a specific subject, say $y_{i 0}$, we are essentially facing a calibration problem, that is, one in which the roles of the explanatory and response variables are interchanged. For details, the reader is referred to Graybill and Iyer (1994, pp. 425-431) among others. For such purpose, we may invert expression (3.3), obtaining

$$
\widehat{\mathrm{PMI}}_{i}\left(y_{i 0}\right)=\frac{\widehat{\beta}^{*}+\widehat{b}_{i}}{\log \left(y_{i 0}\right)-\left(\widehat{\alpha}^{*}+\widehat{a}_{i}^{*}\right)},
$$

where $\widehat{\alpha}^{*}$ and $\widehat{\beta}^{*}$ are the maximum likelihood estimates of $\alpha$ and $\beta$ and $\widehat{a}_{i}^{*}$ and $\widehat{b}_{i}$ are the best linear unbiased predictors of $a_{i}^{*}$ and $b_{i}$ in model (3.3) with the modifications introduced in the covariance structure. The corresponding standard error may be obtained via the Delta Method [see Sen et al. (2009, pp. 211-212), for example]. Since the expression for the this standard error is quite intricate, we present the details in the Appendix.

Estimated PMI and corresponding standard errors for the individual observed values of dif(ARPL) as well as the real (observed) PMI are displayed in Table 3. The individual fitted curves are displayed in Figure 6.

Although some estimates have large standard errors, those corresponding to subjects with 4 or more measurements tend to be smaller than the standard errors obtained by other methods, as reported in Kaliszan et al. (2009).

The predicted PMI corresponding to the second measurements for subjects 8 and 10 and 12 deserve some attention. Subjects 8 and 10 have only two observations and were observed close to 12 hours after death, when hypostasis tend to stabilize, i.e., when the asymptote is reached and this may induces imprecision on the predictions, reflected in the large standard errors. For subject 12, one would expect the value of $\operatorname{dif}(\mathrm{ARPL})$ at the third observation to be closer to that of the second observation.

The $R$ codes for fitting the mixed models (3.1), (3.3) and (3.4), for obtaining the individual predicted PMI and corresponding standard errors as well as for constructing the predicted individual profiles are available at http://www.ime.usp. br/ jmsinger/hypostasis.zip. 
Table 3 Observed values of the response variable [dif(ARPL)] as well as observed and exponential (final) model predicted PMI along with standard errors

\begin{tabular}{|c|c|c|c|c|c|c|c|c|c|}
\hline Subj & $\begin{array}{c}\text { Observed } \\
\text { dif(ARPL) }\end{array}$ & $\begin{array}{l}\text { Real } \\
\text { PMI }\end{array}$ & $\begin{array}{l}\text { Pred } \\
\text { PMI }\end{array}$ & $\begin{array}{l}\text { Std } \\
\text { Error }\end{array}$ & Subj & $\begin{array}{c}\text { Observed } \\
\text { dif(ARPL) }\end{array}$ & $\begin{array}{l}\text { Real } \\
\text { PMI }\end{array}$ & $\begin{array}{l}\text { Pred } \\
\text { PMI }\end{array}$ & $\begin{array}{l}\text { Std } \\
\text { Error }\end{array}$ \\
\hline 1 & 44.0 & 4 & 4.1 & 0.4 & 15 & 35.0 & 4 & 3.6 & 0.8 \\
\hline 2 & 17.0 & 3 & 3.0 & 0.1 & 15 & 48.6 & 5 & 5.0 & 1.0 \\
\hline 3 & 40.7 & 4 & 4.1 & 0.6 & 15 & 64.7 & 6 & 7.2 & 1.0 \\
\hline 3 & 42.6 & 5 & 5.1 & 0.6 & 15 & 70.6 & 7 & 8.3 & 1.0 \\
\hline 3 & 44.5 & 6 & 6.8 & 1.4 & 15 & 72.2 & 8 & 8.6 & 1.0 \\
\hline 3 & 43.7 & 7 & 6.0 & 0.9 & 15 & 70.3 & 9 & 8.2 & 1.0 \\
\hline 4 & 38.5 & 13 & 12.8 & 1.9 & 15 & 74.1 & 10 & 9.1 & 1.0 \\
\hline 4 & 39.1 & 14 & 14.0 & 2.3 & 16 & 44.4 & 12 & 10.8 & 1.2 \\
\hline 5 & 41.8 & 7 & 7.1 & 0.7 & 16 & 48.2 & 13 & 14.9 & 2.0 \\
\hline 6 & 32.9 & 12 & 9.5 & 1.5 & 16 & 47.5 & 14 & 14.0 & 1.5 \\
\hline 6 & 37.7 & 13 & 17.6 & 5.2 & 17 & 34.9 & 12 & 11.2 & 0.7 \\
\hline 7 & 33.2 & 10 & 9.6 & 1.2 & 17 & 36.0 & 13 & 13.0 & 1.0 \\
\hline 7 & 35.1 & 11 & 12.6 & 2.1 & 17 & 36.8 & 14 & 14.7 & 1.6 \\
\hline 7 & 34.0 & 12 & 10.7 & 1.2 & 18 & 39.1 & 8 & 6.6 & 0.6 \\
\hline 8 & 39.9 & 19 & 13.8 & 2.9 & 18 & 47.4 & 9 & 9.9 & 0.5 \\
\hline 8 & 44.5 & 20 & 32.2 & 16.6 & 18 & 50.7 & 10 & 11.9 & 1.2 \\
\hline 9 & 34.6 & 20 & 18.9 & 7.4 & 18 & 51.0 & 11 & 12.1 & 1.3 \\
\hline 10 & 26.8 & 10 & 4.6 & 1.9 & 18 & 52.4 & 12 & 13.2 & 1.9 \\
\hline 10 & 43.0 & 11 & 27.5 & 60.6 & 18 & 52.3 & 13 & 13.7 & 1.8 \\
\hline 11 & 51.0 & 8 & 7.8 & 0.6 & 18 & 53.2 & 14 & 13.9 & 2.3 \\
\hline 11 & 55.4 & 9 & 9.5 & 0.9 & 18 & 52.8 & 15 & 13.6 & 2.0 \\
\hline 12 & 16.8 & 11 & 2.7 & 1.7 & 18 & 53.1 & 16 & 13.9 & 2.2 \\
\hline 12 & 25.6 & 12 & 6.6 & 3.9 & 19 & 47.9 & 8 & 7.6 & 1.1 \\
\hline 12 & 29.1 & 13 & 11.5 & 11.2 & 19 & 52.7 & 9 & 9.4 & 0.9 \\
\hline 13 & 36.1 & 1 & - & - & 19 & 55.9 & 10 & 10.9 & 0.8 \\
\hline 13 & 35.6 & 2 & - & - & 19 & 55.1 & 11 & 10.5 & 0.8 \\
\hline 13 & 36.4 & 3 & - & - & 20 & 40.2 & 11 & 10.9 & 1.1 \\
\hline 13 & 43.1 & 4 & - & - & 20 & 41.4 & 12 & 12.4 & 1.4 \\
\hline 14 & 5.9 & 4 & 1.5 & 0.2 & 20 & 41.6 & 13 & 12.7 & 1.5 \\
\hline 14 & 12.4 & 5 & 2.3 & 0.3 & 21 & 45.9 & 11 & 10.1 & $<0.1$ \\
\hline 14 & 32.9 & 6 & 7.5 & 0.6 & 21 & 48.7 & 12 & 12.1 & $<0.1$ \\
\hline 15 & 21.4 & 3 & 2.6 & 0.7 & 21 & 51.2 & 13 & 14.5 & $<0.1$ \\
\hline
\end{tabular}

\section{Discussion}

In practice, applied statisticians are often faced with the problem of analyzing data generated from observational studies that may have small sample size, be unbalanced, sparse and subject to large variability. Furthermore, the phenomenon under investigation may have certain peculiarities (an asymptote, for example) that must be taken into account in the adopted statistical models. In many cases, there are theoretical subject matter considerations, generally based on differential equations, that indicate the appropriate class of models to be employed in the analysis. Inter- 


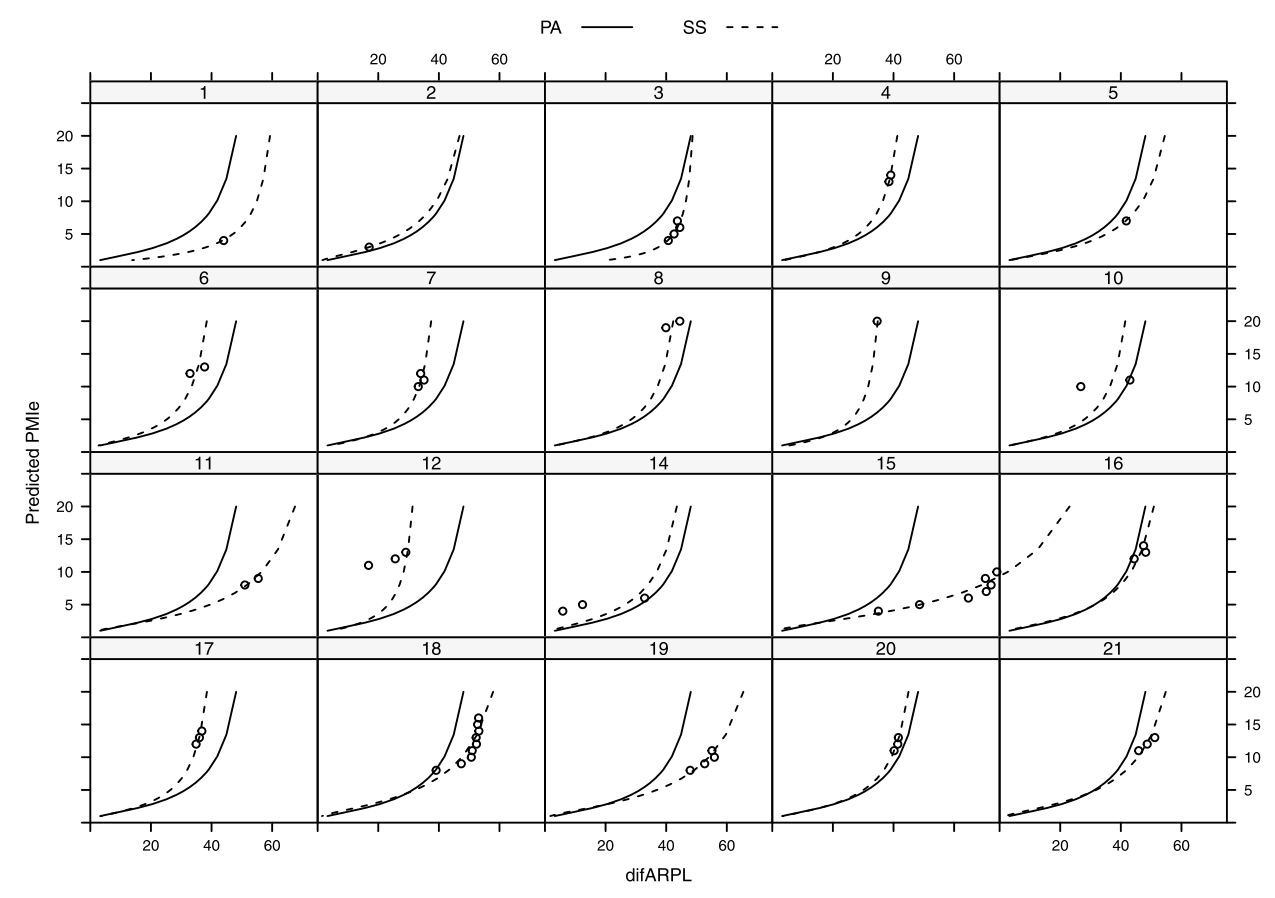

Figure 6 Exponential model estimated individual profiles of the predicted PMI and observed PMI (subjects 13 and third observation of subject 15 excluded); $P A=$ population averaged, $S S=$ subject specific.

pretation of the associated parameters is their most important feature and there is no question that this is the class of models to be considered. There are cases, however, when no such theoretical arguments are available and the analyst must cope with the dilemma of choosing between the numerous non-linear models available in the literature and some more tractable approximate (usually linear) alternative. The data set that motivated this manuscript is a typical example. Even if the results from the analysis based on such models may not be optimal, they are important, specially when the problem under investigation involves new methodologies. The availability of efficient fitting algorithms and diagnostic tools as well as software for their implementation may definitely help in the process of specifying a reasonable model and understanding the data. We believe that development of such tools for non-linear mixed models may be a rewarding direction for research.

Based on the example, we describe an analysis strategy that includes the specification of models with different complexities and evaluate their advantages and caveats regarding convergence of the fitting algorithms, flexibility of interpretation as well as software availability. In general, inclusion of covariates improves the performance of statistical models; however, the sparseness and messy nature of the data in our example with some subjects measured irregularly along time 
and some with a single observation) did not allow a refinement of the analysis by controlling for sex, race and age.

Although attractive, genuine non-linear mixed models like (3.1) may be associated to convergence problems in small samples. In our example, the choice of the initial values for the fitting algorithm was crucial, since in some cases it led to local maxima. We used initial values obtained from fitting von Bertalanffy model with no random effects so that more time was required for analysis. The mixed segmented regression model is appealing because of its simplicity, but requires specialized algorithms when random change-points are included. Linearizable mixed models, on the other hand, may be fitted via standard algorithms (that do not require specification of initial values) and, in general, have good convergence properties. Furthermore, their goodness of fit may be evaluated via well established residual, leverage and local influence analyses and individual estimated curves may be easily constructed.

As in the case of the linearized exponential model (3.2), expressions (3.1) and (3.4) may be inverted to generate predictions of the PMI from a given value of dif(ARPL), say, $y_{i 0}$, yielding, respectively,

$$
\widehat{\mathrm{PMI}}_{i}\left(y_{i 0}\right)=-\frac{1}{\widehat{\delta}+\widehat{d}_{i}} \log \left[1-\frac{y_{i 0}}{\widehat{\gamma}-\widehat{g}_{i}}\right]
$$

and

$$
\widehat{\operatorname{PMI}}_{i}\left(y_{i 0}\right)= \begin{cases}y_{i 0} /\left(\widehat{\kappa}+\widehat{k}_{i}\right) & \text { if } x_{i j} \leq \widehat{\psi}_{i}, \\ \widehat{\psi}_{i} & \text { if } x_{i j}>\widehat{\psi}_{i}\end{cases}
$$

The predicted profiles obtained via the three models are presented in Figures 6, 7 and 8. Because of their non-linear nature, the parameters of both non-linear mixed models have a subject-specific interpretation rather than a population-averaged one and for this reason, are not comparable to those associated to the linearized mixed model. This is why the population averaged profiles are not presented in Figures 7 and 8.

Given the calibration nature of the problem, we may also compare the three models via the difference between the corresponding observed and predicted PMI, displayed in Table 4. The mean squared difference between the observed and predicted PMI for the exponential, von Bertalanffy and segmented regression models are, respectively, 11.01, 13.53 and 5.48, indicating that the segmented regression model may be preferable for predicting the PMI associated to the sample subjects.

If, however, the objective is to predict the behaviour of a new subject, the exponential model is certainly more convenient. In this context, two cases may be considered

(i) no measurements of $\operatorname{dif}(\mathrm{ARPL})$ are available for this subject; 


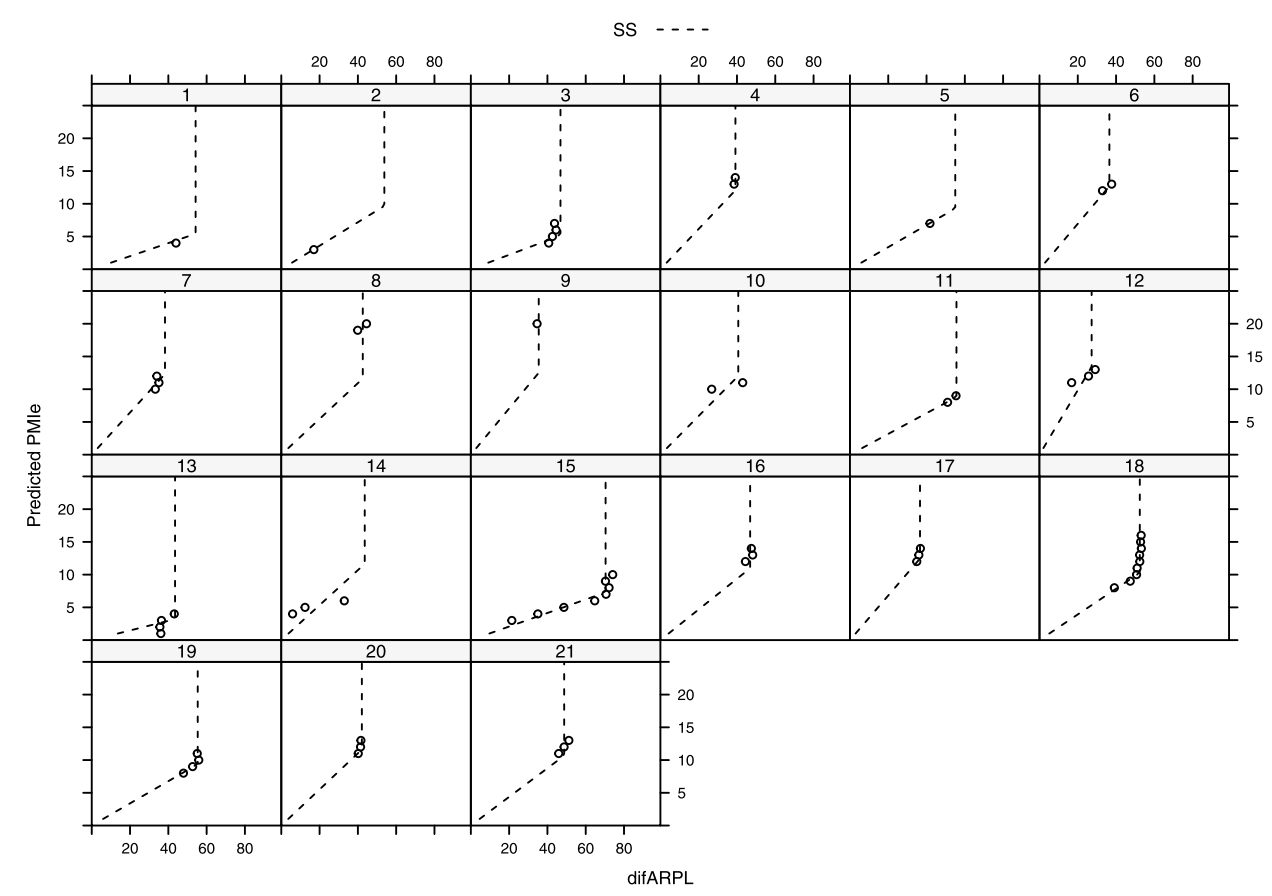

Figure 7 Segmented regression model estimated individual profiles of the predicted PMI and observed PMI; SS = subject specific.

(ii) at least one measurement of $\operatorname{dif(ARPL)~is~available~for~this~subject.~}$

In the first case, prediction of the PMI must be carried out via the estimates of the population averaged parameters [(A.2) in the Appendix with $\boldsymbol{\Omega}$ and $\Gamma$ replaced by their estimates]. Note that this option may not be considered when the analysis is carried out via the non-liner models. In the second case, one may consider two approaches:

(a) use the estimates of both the population averaged and subject specific parameters [(A.2)-(A.3) in the Appendix with $\boldsymbol{\Omega}$ and $\Gamma$ replaced by their estimates] with the addition of new rows to $\mathbf{X}$ and $\mathbf{Z}$ corresponding to the observed times for the new subject and to $\mathbf{y}$ with the associated observed values of $\operatorname{dif}($ ARPL);

(b) refit the entire model with the inclusion of the new subject data.

Here we note that although we used the AIC only as an additional criterion to help in the choice of an appropriate model (within each class) there may be some concern as to whether a marginal (which we adopted) or a conditional version should be employed as discussed in Vaida and Blanchard (2005) or Liang et al. (2008). In cases where the interest lies in the population averaged parame- 


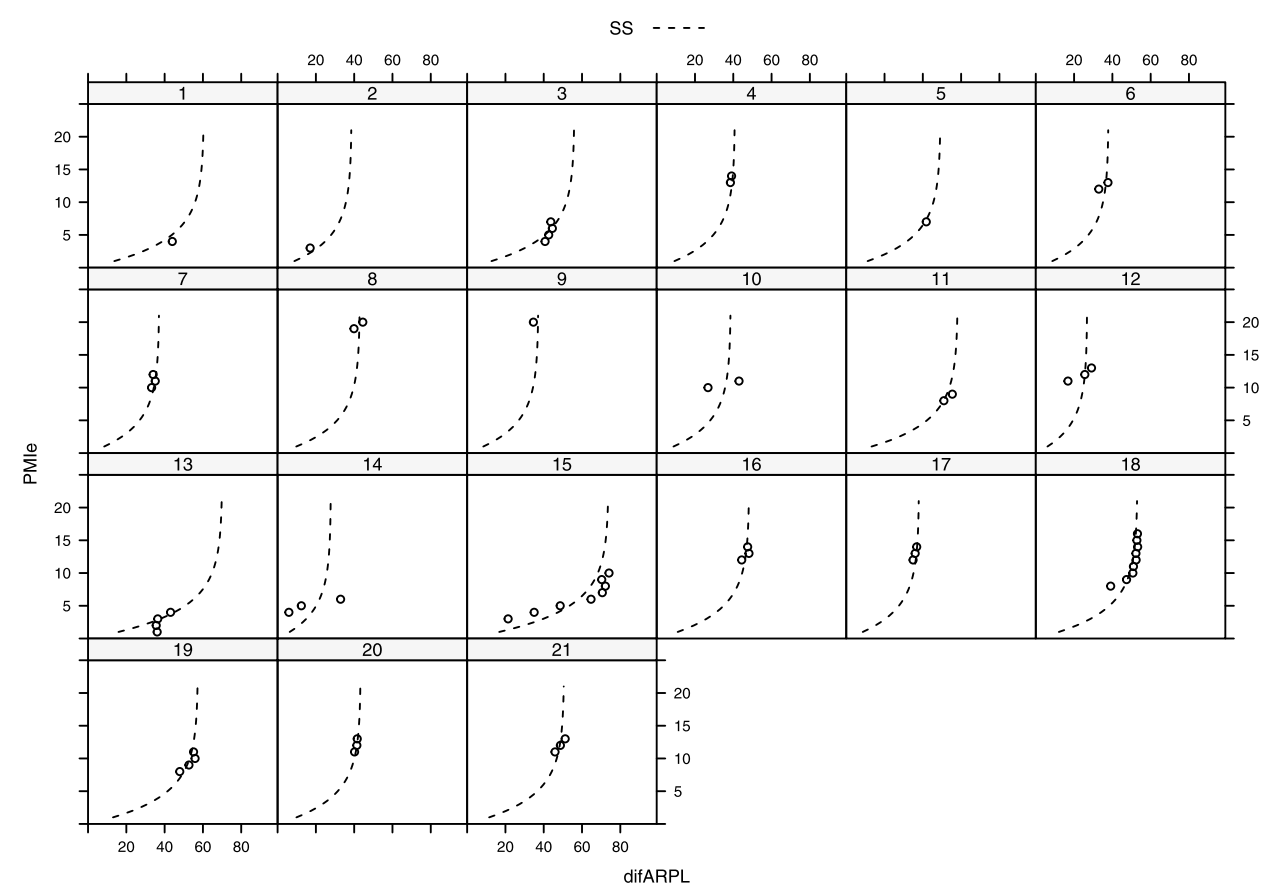

Figure 8 von Bertalanffy model estimated individual profiles of the predicted PMI and observed $P M I ; S S=$ subject specific.

ters, these authors suggest that a marginal AIC should be considered; otherwise, if the interest lies in the subject specific parameters, the conditional AIC is preferred. Since depending on the availability of information for a new subject, we may be interested in either population averaged or subject specific inferences, it is difficult to choose between the two approaches. Furthermore, these authors have developed the conditional AIC methodology for models that are simpler than the ones we considered. Nevertheless, we feel that this might open an interesting field for further research.

Finally, we note that if considered for determination of PMI within the first 12 hours after death and provided 4 or more measurements may be obtained on a subject, the proposed method may generate better results (with slightly smaller margins of errors) than the standard ones, like those based on body cooling and drying out of the cornea as described by Kaliszan et al. (2009). We recognize the limitations of the motivating study, conducted under non-ideal conditions and note that the proposed technique for evaluation of PMI requires further investigation before it may be employed in practical applications. All the available methods have limitations so that their concomitant use may generate more precise and useful results. 
Table 4 Predicted PMI

\begin{tabular}{|c|c|c|c|c|c|c|c|c|c|}
\hline \multirow[b]{2}{*}{ Subject } & \multirow[b]{2}{*}{ Real PMI } & \multicolumn{3}{|c|}{ Predicted PMI } & \multirow[b]{2}{*}{ Subject } & \multirow[b]{2}{*}{ Real PMI } & \multicolumn{3}{|c|}{ Predicted PMI } \\
\hline & & Exp & Bert & Seg & & & Exp & Bert & Seg \\
\hline 1 & 4.0 & 4.1 & 5.1 & 4.4 & 15 & 4.0 & 3.6 & 2.5 & 3.6 \\
\hline 2 & 3.0 & 3.0 & 2.3 & 3.1 & 15 & 5.0 & 5.0 & 4.2 & 5.0 \\
\hline 3 & 4.0 & 4.1 & 5.1 & 4.5 & 15 & 6.0 & 7.2 & 8.1 & 6.7 \\
\hline 3 & 5.0 & 5.1 & 5.6 & 4.7 & 15 & 7.0 & 8.3 & 11.8 & 7.3 \\
\hline 3 & 6.0 & 6.8 & 6.2 & 5.2 & 15 & 8.0 & 8.6 & 13.9 & 7.3 \\
\hline 3 & 7.0 & 6.0 & 5.9 & 5.2 & 15 & 9.0 & 8.2 & 11.5 & 7.3 \\
\hline 4 & 13.0 & 12.8 & 11.3 & 12.1 & 15 & 10.0 & 9.1 & 20.4 & 7.3 \\
\hline 4 & 14.0 & 14.0 & 12.5 & 12.1 & 16 & 12.0 & 10.8 & 9.9 & 11.0 \\
\hline 5 & 7.0 & 7.1 & 7.4 & 7.1 & 16 & 13.0 & 14.9 & 20.6 & 11.0 \\
\hline 6 & 12.0 & 9.5 & 8.0 & 11.4 & 16 & 14.0 & 14.0 & 15.6 & 11.0 \\
\hline 6 & 13.0 & 17.6 & 18.5 & 12.7 & 17 & 12.0 & 11.2 & 9.9 & 11.9 \\
\hline 7 & 10.0 & 9.6 & 8.9 & 10.7 & 17 & 13.0 & 13.0 & 11.6 & 12.5 \\
\hline 7 & 11.0 & 12.6 & 11.3 & 11.3 & 17 & 14.0 & 14.7 & 13.5 & 12.5 \\
\hline 7 & 12.0 & 10.7 & 9.7 & 10.9 & 18 & 8.0 & 6.6 & 5.3 & 7.7 \\
\hline 8 & 19.0 & 13.8 & 10.5 & 11.6 & 18 & 9.0 & 9.9 & 8.8 & 9.3 \\
\hline 8 & 20.0 & 32.2 & - & 11.6 & 18 & 10.0 & 11.9 & 12.1 & 9.9 \\
\hline 9 & 20.0 & 18.9 & 10.7 & 12.5 & 18 & 11.0 & 12.1 & 12.6 & 10.3 \\
\hline 10 & 10.0 & 4.6 & 4.7 & 7.9 & 18 & 12.0 & 13.2 & 16.3 & 10.3 \\
\hline 10 & 11.0 & 27.5 & - & 12.7 & 18 & 13.0 & 13.7 & 15.9 & 10.3 \\
\hline 11 & 8.0 & 7.8 & 8.2 & 8.1 & 18 & 14.0 & 13.9 & 24.5 & 10.3 \\
\hline 11 & 9.0 & 9.5 & 11.5 & 8.8 & 18 & 15.0 & 13.6 & 18.6 & 10.3 \\
\hline 12 & 11.0 & 2.7 & 3.9 & 8.3 & 18 & 16.0 & 13.9 & 22.1 & 10.3 \\
\hline 12 & 12.0 & 6.6 & 12.2 & 12.6 & 19 & 8.0 & 7.6 & 7.1 & 8.1 \\
\hline 12 & 13.0 & 11.5 & - & 14.3 & 19 & 9.0 & 9.4 & 9.8 & 8.9 \\
\hline 13 & 1.0 & - & 2.8 & 2.7 & 19 & 10.0 & 10.9 & 13.8 & 9.4 \\
\hline 13 & 2.0 & - & 2.8 & 2.6 & 19 & 11.0 & 10.5 & 12.3 & 9.4 \\
\hline 13 & 3.0 & - & 2.9 & 2.7 & 20 & 11.0 & 10.9 & 10.3 & 11.1 \\
\hline 13 & 4.0 & - & 3.7 & 3.2 & 20 & 12.0 & 12.4 & 12.1 & 11.6 \\
\hline 14 & 4.0 & 1.5 & 1.0 & 1.6 & 20 & 13.0 & 12.7 & 12.5 & 11.6 \\
\hline 14 & 5.0 & 2.3 & 2.4 & 3.3 & 21 & 11.0 & 10.1 & 9.4 & 10.7 \\
\hline 14 & 6.0 & 7.5 & - & 8.8 & 21 & 12.0 & 12.1 & 12.7 & 10.7 \\
\hline 15 & 3.0 & 2.6 & 1.4 & 2.2 & 21 & 13.0 & 14.5 & - & 10.7 \\
\hline
\end{tabular}

Exp: exponential model.

Bert: Bertalanffy model.

Seg: segmented regression model.

\section{Appendix}

\section{Details for the linear mixed model (3.3)}

Consider the linear mixed model

$$
\mathbf{y}_{i}=\mathbf{X}_{i} \boldsymbol{\beta}+\mathbf{Z}_{i} \mathbf{b}_{i}+\mathbf{e}_{i}, \quad i=1, \ldots, n,
$$


where $\mathbf{y}_{i}=\left(y_{i 1}, \ldots, y_{i m_{i}}\right)^{\top}$ is a $m_{i} \times 1$ vector of observations for the $i$ th subject, $\boldsymbol{\beta}=\left(\beta_{1}, \ldots, \beta_{p}\right)^{\top}$ is a $p \times 1$ vector of unknown population parameters, $\mathbf{X}_{i}$ is a $m_{i} \times p$ known specification matrix corresponding to the fixed effects, $\mathbf{b}_{i}=\left(b_{i 1}, \ldots, b_{i q}\right)^{\top}$ is a $q \times 1$ vector of unobservable random elements, $\mathbf{Z}_{i}$ is a $m_{i} \times q$ known specification matrix corresponding to the random effects and $\mathbf{e}_{i}=\left(e_{i 1}, \ldots, e_{i m_{i}}\right)^{\top}$ is an $m_{i} \times 1$ vector of random errors. Assume that the $\mathbf{b}_{i}$ and the $\mathbf{e}_{i}$ are all uncorrelated and are such that $\mathbb{E}\left(\mathbf{b}_{i}\right)=\mathbf{0}, \mathbb{V}\left(\mathbf{b}_{i}\right)=\mathbf{G}, \mathbb{E}\left(\mathbf{e}_{i}\right)=\mathbf{0}$, $\mathbb{V}\left(\mathbf{e}_{i}\right)=\mathbf{R}_{i}$, where $\mathbf{G}=\mathbf{G}(\boldsymbol{\theta})$ and $\mathbf{R}_{i}=\mathbf{R}_{i}(\boldsymbol{\theta})$ are, respectively, $q \times q$ and $m_{i} \times m_{i}$ positive-definite symmetric matrices depending on an $t \times 1$ covariance parameter vector $\boldsymbol{\theta}$, not functionally related to $\boldsymbol{\beta}$.

Letting $\mathbf{y}=\left(\mathbf{y}_{1}^{\top}, \ldots, \mathbf{y}_{n}^{\top}\right)^{\top}, \mathbf{X}=\left(\mathbf{X}_{1}^{\top}, \ldots, \mathbf{X}_{n}^{\top}\right)^{\top}, \mathbf{Z}=\bigoplus_{i=1}^{n} \mathbf{Z}_{i}, \mathbf{b}=\left(\mathbf{b}_{1}^{\top}, \ldots\right.$, $\left.\mathbf{b}_{n}^{\top}\right)^{\top}$ and $\mathbf{e}=\left(\mathbf{e}_{1}^{\top}, \ldots, \mathbf{e}_{n}^{\top}\right)^{\top}$, we can write model (A.1) more compactly as

$$
\mathbf{y}=\mathbf{X} \boldsymbol{\beta}+\mathbf{Z b}+\mathbf{e} .
$$

This implies that $\mathbb{E}(\mathbf{y})=\mathbf{X} \boldsymbol{\beta}$ and $\mathbb{V}(\mathbf{y})=\mathbf{\Omega}=\mathbf{Z} \boldsymbol{\Gamma} \mathbf{Z}^{\top}+\mathbf{R}$ where $\boldsymbol{\Gamma}=\mathbf{I}_{n} \otimes \mathbf{G}$ and $\mathbf{R}=\bigoplus_{i=1}^{n} \mathbf{R}_{i}$.

Based on the joint distribution of $\mathbf{y}$ and $\mathbf{b}$ and assuming that the covariance matrices $\boldsymbol{\Gamma}$ and $\mathbf{R}$ are known, best linear unbiased estimators (BLUE) of the fixed effects $\boldsymbol{\beta}$ and best linear predictors (BLUP) of the random effects $\mathbf{b}_{i}$ may be obtained as the solutions to the Henderson (1975) equations

$$
\left(\begin{array}{cc}
\mathbf{X}^{\top} \mathbf{R}^{-1} \mathbf{X} & \mathbf{X}^{\top} \mathbf{R}^{-1} \mathbf{Z} \\
\mathbf{Z}^{\top} \mathbf{R}^{-1} \mathbf{X} & \mathbf{X}^{\top} \mathbf{R}^{-1} \mathbf{Z}+\Gamma^{-1}
\end{array}\right)\left(\begin{array}{c}
\widehat{\boldsymbol{\beta}} \\
\widehat{\mathbf{b}}
\end{array}\right)\left(\begin{array}{l}
\mathbf{X}^{\top} \mathbf{R}^{-1} \mathbf{y} \\
\mathbf{Z}^{\top} \mathbf{R}^{-1} \mathbf{y}
\end{array}\right)
$$

namely,

$$
\begin{aligned}
& \widehat{\boldsymbol{\beta}}=\left(\mathbf{X}^{\top} \boldsymbol{\Omega}^{-1} \mathbf{X}\right)^{-1} \mathbf{X}^{\top} \boldsymbol{\Omega}^{-1} \mathbf{y}, \\
& \widehat{\mathbf{b}}=\boldsymbol{\Gamma} \mathbf{Z}^{\top} \boldsymbol{\Omega}^{-1}(\mathbf{y}-\mathbf{X} \widehat{\boldsymbol{\beta}})=\boldsymbol{\Gamma} \mathbf{Z}^{\top} \mathbf{Q} \mathbf{y},
\end{aligned}
$$

where $\mathbf{Q}=\boldsymbol{\Omega}^{-1}-\boldsymbol{\Omega}^{-1} \mathbf{X}\left(\mathbf{X}^{\top} \boldsymbol{\Omega}^{-1} \mathbf{X}\right)^{-1} \mathbf{X}^{\top} \boldsymbol{\Omega}^{-1}$. This implies that

$$
\begin{aligned}
\mathbb{E}(\widehat{\boldsymbol{\beta}}) & =\boldsymbol{\beta}, & \mathbb{V}(\widehat{\boldsymbol{\beta}})=\left(\mathbf{X}^{\top} \mathbf{\Omega}^{-1} \mathbf{X}\right)^{-1}, \\
\mathbb{E}(\widehat{\mathbf{b}}) & =\mathbf{0}, & \mathbb{V}(\widehat{\mathbf{b}})=\boldsymbol{\Gamma} \mathbf{Z}^{\top} \mathbf{Q Z} \boldsymbol{\Gamma}, \\
\mathbb{C o v}(\widehat{\boldsymbol{\beta}}, \widehat{\mathbf{b}}) & =\mathbf{0} . &
\end{aligned}
$$

As noted by Harville (1976) and Laird and Ware (1982) among others, the variance of $\widehat{\mathbf{b}}$ does not take the variability of the random effects $\mathbf{b}$ into account so that for prediction purposes, it is more appropriate to use

$$
\begin{aligned}
\mathbb{V}(\widehat{\mathbf{b}}-\mathbf{b}) & =\boldsymbol{\Gamma}-\boldsymbol{\Gamma} \mathbf{Z}^{\top} \mathbf{Q Z} \boldsymbol{\Gamma}, \\
\operatorname{Cov}[\widehat{\boldsymbol{\beta}},(\widehat{\mathbf{b}}-\mathbf{b})] & =-\left(\mathbf{X}^{\top} \boldsymbol{\Omega}^{-1} \mathbf{X}\right)^{-1} \mathbf{X}^{\top} \boldsymbol{\Omega}^{-1} \mathbf{Z} \boldsymbol{\Gamma} .
\end{aligned}
$$

In practice, we replace the unknown elements of $\mathbf{G}$ and $\mathbf{R}$ with estimates. 


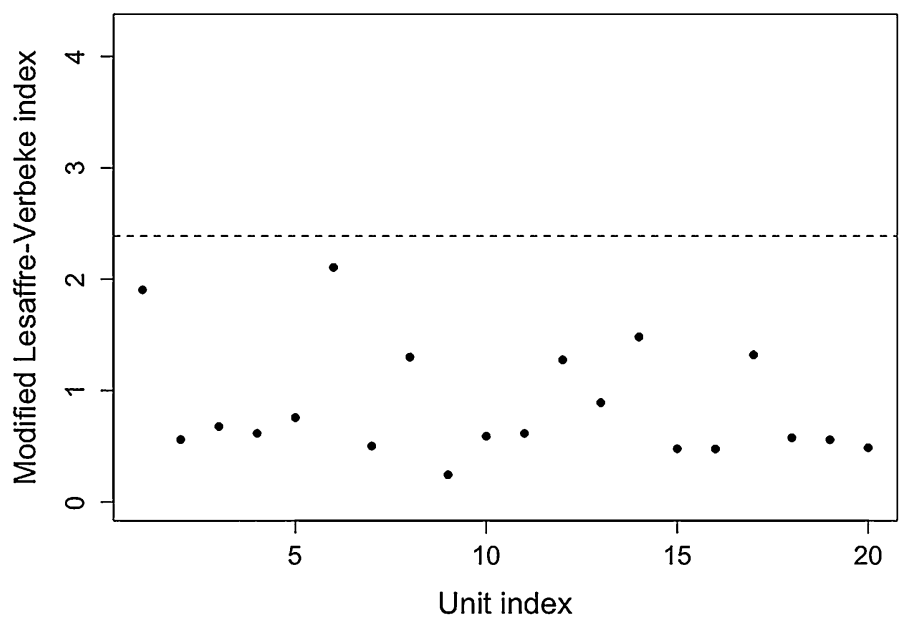

Figure A.1 Plot of the modified Lesaffre-Verbeke measure for the final exponential model (with different variance components for subjects 10,12,14 and 15 after excluding subject 13 and the first observation of subject 15$)$. Dashed line: 3 rd quartile +1.5 interquartile range.

Now consider predictors of the form $\widehat{\mathbf{P}}=\mathbf{L} \widehat{\boldsymbol{\beta}}+\mathbf{D} \widehat{\mathbf{b}}=\mathbf{K} \widehat{\mathbf{B}}$ where $\mathbf{K}=(\mathbf{L D})$ and $\widehat{\mathbf{B}}=\left(\widehat{\boldsymbol{\beta}}^{\top}, \widehat{\mathbf{b}}^{\top}\right)^{\top}$. Then, letting $\mathbf{V}_{\widehat{\mathbf{B}}}=\mathbb{V}\left[\widehat{\boldsymbol{\beta}}^{\top},(\widehat{\mathbf{b}}-\mathbf{b})^{\top}\right]^{\top}$, it follows that $\mathbf{V}_{\widehat{\mathbf{P}}}=$ $\mathbb{V}(\widehat{\mathbf{P}})=\mathbf{K} \mathbf{V}_{\widehat{\mathbf{B}}} \mathbf{K}^{\top}$.

For the hypostasis data, $\widehat{\boldsymbol{\beta}}=\left(\widehat{\alpha}^{*}, \widehat{\beta}^{*}\right)^{\top}, \widehat{\mathbf{b}}=\bigoplus_{i=1}^{21}\left(\widehat{a}_{i}^{*}, \widehat{b}_{i}\right)^{\top} \mathbf{1}_{42}$, where $\mathbf{1}_{r}$ denotes a vector of dimension $r$ with all elements equal to $1, \mathbf{L}=\mathbf{1}_{21} \otimes \mathbf{I}_{2}$, with $\mathbf{I}_{r}$ denoting the identity matrix of dimension $r$ and $\mathbf{D}=\mathbf{I}_{42}$. This implies that $\widehat{\mathbf{P}}=\bigoplus_{i=1}^{21} \widehat{\mathbf{P}}_{i} \mathbf{1}_{42}$ with $\widehat{\mathbf{P}}_{i}=\left(\widehat{\alpha}_{i}^{*}, \widehat{\beta}_{i}^{*}\right)^{\top}, \widehat{\alpha}_{i}^{*}=\widehat{\alpha}^{*}+\widehat{a}_{i}^{*}$ and $\widehat{\beta}_{i}^{*}=\widehat{\beta}^{*}+\widehat{b}_{i}$. The variance of $\widehat{\mathbf{P}}_{i}$ corresponds to the $2 \times 2$ block matrix associated to the $i$ th subject in $\mathbf{V}_{\widehat{\mathbf{p}}}$.

Recalling (4.1) and letting $\widehat{\mathrm{PMI}}_{i}\left(y_{i 0}\right)=g\left(\widehat{\alpha}_{i}^{*}, \widehat{\beta}_{i}^{*}\right)$, it follows that

$$
\begin{aligned}
& \partial g\left(\widehat{\alpha}_{i}^{*}, \widehat{\beta}_{i}^{*}\right) / \partial \widehat{\alpha}_{i}^{*}=-\widehat{\beta}_{i}^{*} /\left[\log \left(y_{i 0}\right)-\widehat{\alpha}_{i}^{*}\right]^{2} \text { and } \\
& \partial g\left(\widehat{\alpha}_{i}^{*}, \widehat{\beta}_{i}^{*}\right) / \partial \widehat{\beta}_{i}^{*}=1 /\left[\log \left(y_{i 0}\right)-\widehat{\alpha}_{i}^{*}\right] .
\end{aligned}
$$

Letting

$$
\dot{\mathbf{g}}\left(\widehat{\alpha}_{i}^{*}, \widehat{\beta}_{i}^{*}\right)=\left\{-\widehat{\beta}_{i}^{*} /\left[\log \left(y_{i 0}\right)-\widehat{\alpha}_{i}^{*}\right]^{2}, 1 /\left[\log \left(y_{i 0}\right)-\widehat{\alpha}_{i}^{*}\right]\right\}^{\top}
$$

we may use the Delta method to compute

$$
\mathbb{V}\left[\widehat{\operatorname{PMI}}_{i}\left(y_{i 0}\right)\right]=\dot{\mathbf{g}}\left(\widehat{\alpha}_{i}^{*}, \widehat{\beta}_{i}^{*}\right)^{\top} \mathbb{V}\left(\widehat{\mathbf{P}}_{i}\right) \dot{\mathbf{g}}\left(\widehat{\alpha}_{i}^{*}, \widehat{\beta}_{i}^{*}\right) .
$$

\section{Algorithm for fitting the mixed segmented regression model (3.4)}

Details on an adaptation of the algorithm proposed by Muggeo et al. (2014) for the specific case under investigation are presented below. For notational simplicity, we 


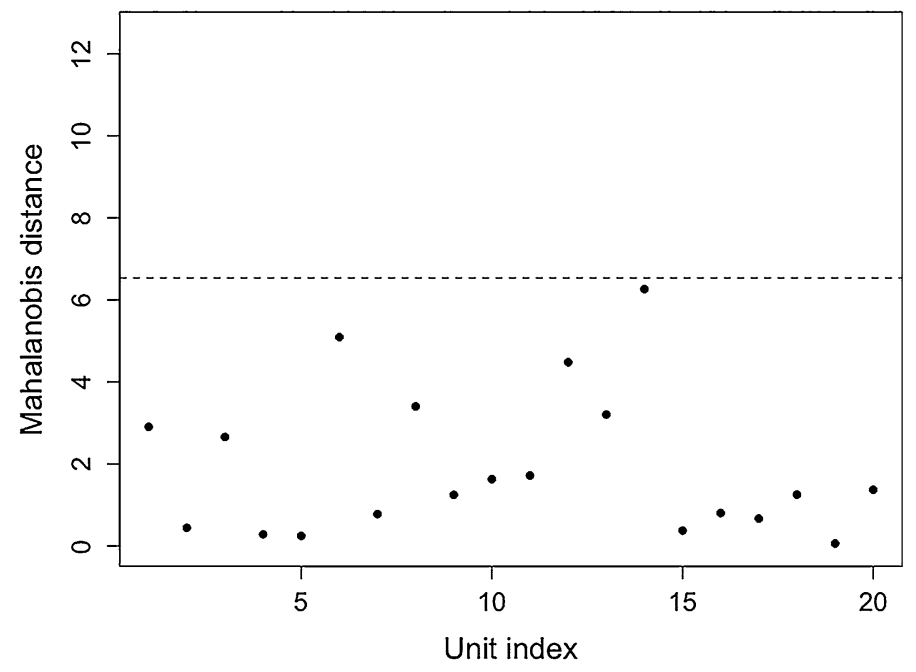

Figure A.2 Mahalanobis distance for the final exponential model (with different variance components for subjects 10,12,14 and 15 after excluding subject 13 and the first observation of subject 15). Dashed line: 3 rd quartile +1.5 interquartile range.

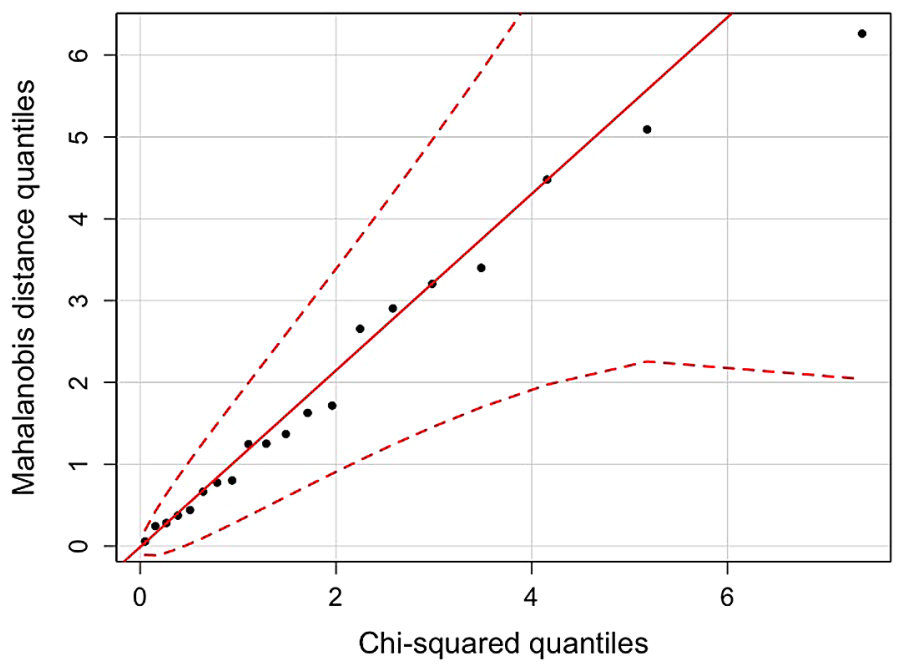

Figure A.3 Mahalanobis distance QQ plot for the final exponential model (with different variance components for subjects 10,12, 14 and 15 after excluding subject 13 and the first observation of subject 15$)$. Dashed line: 3 rd quartile +1.5 interquartile range.

re-express model (3.4) as

$$
y_{i j}=\kappa_{i} f\left(x_{i j}, \psi_{i}\right)+e_{i j},
$$



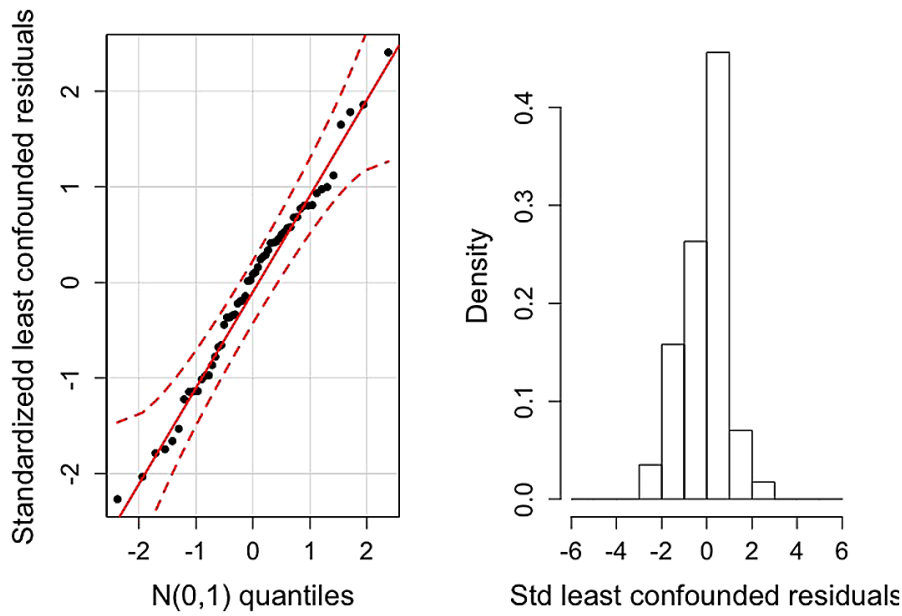

Figure A.4 Least confounded residual QQ plot and histogram for the final exponential model (with different variance components for subjects 10,12,14 and 15 after excluding subject 13 and the first observation of subject 15$)$.

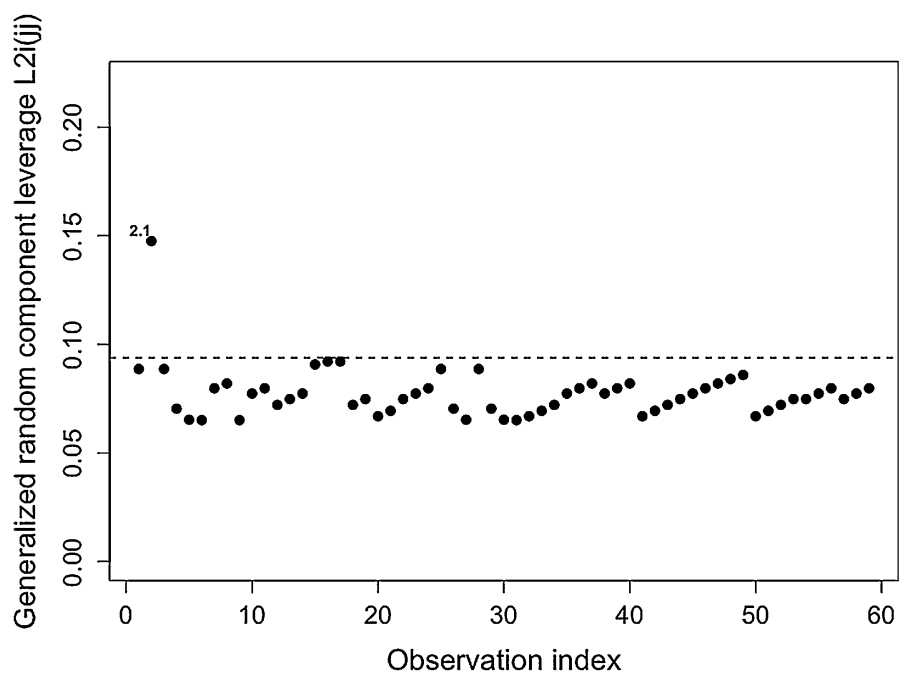

Figure A.5 Plot of the generalized random component leverage for the final exponential model (with different variance components for subjects 10,12, 14 and 15 after excluding subject 13 and the first observation of subject 15$)$. Dashed line: 3 rd quartile +1.5 interquartile range.

where $f\left(x_{i j}, \psi_{i}\right)=\psi_{i}+\left(x_{i j}-\psi_{i}\right) I_{\left\{x_{i j} \leq \psi_{i}\right\}}$. To restrict the possible values of $\psi_{i}$ to the interval $\left(a_{1}, a_{2}\right)$ where where $a_{1}$ and $a_{2}$ are, respectively, the minimum and maximum observed values of $x_{i j}$, we consider the reparametrization

$$
\psi_{i}=\psi_{i}\left(\lambda_{i}\right)=\frac{a_{1}+a_{2} \exp \left(\lambda_{i}\right)}{1+\exp \left(\lambda_{i}\right)}
$$


We proceed with the first order Taylor expansion

$$
f\left[x_{i j}, \psi_{i}\left(\lambda_{i}\right)\right] \approx f\left[x_{i j}, \psi\left(\widehat{\lambda}_{i}\right)\right]+\left.\left(\lambda_{i}-\widehat{\lambda}_{i}\right) \frac{\partial f\left(x_{i j}, \psi_{i}\right)}{\partial \lambda_{i}}\right|_{\lambda_{i}=\hat{\lambda}_{i}} .
$$

Observing that $\partial f\left(x_{i j}, \psi_{i}\right) / \partial \psi_{i}=1-I_{\left\{x_{i j} \leq \psi_{i}\right\}}=h_{i j}\left(\lambda_{i}\right)$ and that $\partial \psi\left(\lambda_{i}\right) / \partial \lambda_{i}=$ $\left(a_{2}-a_{1}\right) \exp \left(\lambda_{i}\right) /\left[1+\exp \left(\lambda_{i}\right)\right]^{2}=g_{i}\left(\lambda_{i}\right)$, we may write

$$
\left.\frac{\partial f\left[x_{i j}, \psi_{i}\left(\lambda_{i}\right]\right.}{\partial \lambda_{i}}\right|_{\lambda_{i}=\widehat{\lambda}_{i}}=h_{i j}\left(\widehat{\lambda}_{i}\right) g_{i}\left(\widehat{\lambda}_{i}\right) \text {. }
$$

Therefore, we have

$$
\begin{aligned}
y_{i j} & \approx \kappa_{i}\left\{\psi\left(\widehat{\lambda}_{i}\right)+\left[x_{i j}-\psi\left(\widehat{\lambda}_{i}\right)\right] I_{\left\{x_{i j} \leq \psi_{i}\left(\widehat{\lambda}_{i}\right)\right\}}+\left(\lambda_{i}-\widehat{\lambda}_{i}\right) h_{i j}\left(\widehat{\lambda}_{i}\right) g_{i}\left(\widehat{\lambda}_{i}\right)\right\} \\
& \approx \kappa_{i} u_{i j}\left(\widehat{\lambda}_{i}\right)+\widehat{\kappa}_{i}\left(\lambda_{i}-\widehat{\lambda}_{i}\right) h_{i j}\left(\widehat{\lambda}_{i}\right) g_{i}\left(\widehat{\lambda}_{i}\right) \\
& \approx \kappa_{i} u_{i j}\left(\widehat{\lambda}_{i}\right)+\lambda_{i} \widehat{\kappa}_{i} h_{i j}\left(\widehat{\lambda}_{i}\right) g_{i}\left(\widehat{\lambda}_{i}\right)-\widehat{\kappa}_{i} \widehat{\lambda}_{i} h_{i j}\left(\widehat{\lambda}_{i}\right) g_{i}\left(\widehat{\lambda}_{i}\right)
\end{aligned}
$$

where $u_{i j}\left(\widehat{\lambda}_{i}\right)=\psi\left(\widehat{\lambda}_{i}\right)+\left[x_{i j}-\psi\left(\widehat{\lambda}_{i}\right)\right] I_{\left\{x_{i j} \leq \psi\left(\widehat{\lambda}_{i}\right)\right\}}$. We may re-express the model as

$$
y_{i j}^{*}=y_{i j}+\widehat{\kappa}_{i} \widehat{\lambda}_{i} h_{i j}\left(\widehat{\lambda}_{i}\right) g_{i}\left(\widehat{\lambda}_{i}\right)=\kappa_{i} u_{i j}\left(\widehat{\lambda}_{i}\right)+\lambda_{i} \widehat{\kappa}_{i} h_{i j}\left(\widehat{\lambda}_{i}\right) g_{i}\left(\widehat{\lambda}_{i}\right)+e_{i j},
$$

where $\widehat{\kappa}_{i} \widehat{\lambda}_{i} h_{i j}\left(\widehat{\lambda}_{i}\right) g_{i}\left(\widehat{\lambda}_{i}\right)$ is considered as an offset. This suggests the following algorithm to obtain the maximum likelihood estimators of the parameters and predictors of the random effects:

Step 1: Set $r=0$ and $\psi_{i}^{(0)}=\psi^{(0)}$.

Step 2: Fit the linear mixed model $y_{i j}=\psi_{i}^{(0)}+\kappa_{i}\left(x_{i j}-\psi_{i}^{(0)}\right) I_{\left\{x_{i j} \leq \psi_{i}^{(0)}\right\}}+e_{i j}$ to obtain $\kappa_{i}^{(0)}$.

Step 3: Compute $\lambda_{i}^{(r)}=\log \left[\left(\psi_{i}^{(r)}-a_{1}\right) /\left(a_{2}-\psi_{i}^{(r)}\right)\right], h_{i j}^{(r)}=I_{\left\{x_{i j}-\psi_{i}^{(r)}\right\}}, g_{i}^{(r)}=$ $\left(a_{2}-a_{1}\right) \exp \left(\lambda_{i}^{(r)}\right) /\left[1+\exp \left(\lambda_{i}^{(r)}\right)\right]^{2}, y_{i j}^{(r)}=y_{i j}-\kappa_{i}^{(r)} \lambda_{i}^{(r)} h_{i j}^{(r)} g_{i}^{(r)}$.

Step 4: Fit the linear mixed model $y_{i j}^{(r)}=\kappa_{i}\left(x_{i j}-\psi_{i}^{(r)}\right) h_{i j}^{(r)}+\kappa_{i}^{(r)} \lambda_{i} g_{i}^{(r)} h_{i j}^{(r)}+$ $e_{i j}$ to obtain $\kappa_{i}^{(r+1)}, \lambda_{i}^{(r+1)}$ and $\psi_{i}^{(r+1)}=\left[a_{1}+a_{2} \exp \left(\lambda_{i}^{(r+1)}\right)\right] /\left[1+\exp \left(\lambda_{i}^{(r+1)}\right)\right]$.

Step 5: Verify if some convergence criterion is satisfied. If yes, stop and $\kappa_{i}^{(r+1)}$, and $\psi_{i}^{(r+1)}$ will provide the required estimates. Otherwise, set $r=r+1$ and return to Step 3 .

\section{Acknowledgments}

This research received financial support from Conselho Nacional de Desenvolvimento Científico e Tecnológico (CNPq, Grant 3304126/2015-2) and Fundação de Amparo à Pesquisa do Estado de São Paulo (FAPESP, Grant 2013/21728-2), Brazil. The authors are grateful to two anonymous referees for their enlightening and constructive comments. 


\section{References}

Davidian, M. and Giltinan, D. M. (2003). Nonlinear models for repeated measurement data: An overview and update. Journal of Agricultural, Biological, and Environmental Statistics 8, 387419.

Demidenko, E. (2013). Mixed Models: Theory and Applications with R, 2nd ed. New York: Wiley.

Dolinak, D., Matshes, E. and Lew, E. O. (2005). Forensic Pathology: Principles and Practice. New York: Elsevier Academic Press.

Fávero, F. (1991) Medicina Legal, 12a ed. Belo Horizonte: Villa Rica.

Fitzmaurice, G. M., Laird, N. M. and Ware, J. H. (2011). Applied Longitudinal Analysis, 2nd ed. New York: Wiley.

Graybill, F. A. and Iyer, H. K. (1994). Regression Analysis: Concepts and Applications. Belmont, CA: Duxbury Press.

Harville, D. (1976). Extension of the Gauss-Markov theorem to include the estimation of random effects. The Annals of Statistics 4, 384-395.

Henderson, C. R. (1975). Best linear unbiased estimation and prediction under a selection model. Biometrics 31, 423-447.

Ishida, M., Gonoi, W., Hagiwara, K., Takazawa, Y., Akahane, M., Fukayama, M. and Ohtomo, K. (2011). Hypostasis in the heart and great vessels of non-traumatic in-hospital death cases on postmortem computed tomography: Relationship to antemortem blood tests. Legal Medicine (Tokyo) 13, 280-285.

Kaliszan, M., Hauser, R. and Kernbach-Wighton, G. (2009). Estimation of the time of death based on the assessment of post mortem processes with emphasis on body cooling. Legal Medicine (Tokyo) 11, 111-117.

Knight, B. (1991). Forensic Pathology, 1st ed. London: Edward Arnold.

Laird, N. M. and Ware, J. H. (1982). Random-effects models for longitudinal data. Biometrics 38, 963-974.

Levy, A. D., Harcke, H. T. and Mallak, C. T. (2010). Postmortem imaging: MDCT features of postmortem change and decomposition. American Journal of Forensic Medicine and Pathology 31, $12-17$.

Liang, H., Wu, H. and Zou, G. (2008). A note on the conditional AIC for linear mixed-effects models. Biometrika 95, 773-778.

Lindsey, J. K. (2004). Statistical Analysis of Stochastic Processes in Time. Cambridge: Cambridge University Press.

Lindstrom, M. J. and Bates, D. M. (1990). Nonlinear mixed effects models for repeated measures data. Biometrics 46, 673-687.

Muggeo, V. M. R., Atkins, D. C., Gallop, R. J. and Dimidjian, S. (2014). Segmented mixed models with random changepoints: A maximum likelihood approach with application to treatment for depression study. Statistical Modelling 14, 293-313.

Pinheiro, J., Bates, D. M., DebRoy, S. Sarkar, D. and R Core Team (2014). nlme: Linear and Nonlinear Mixed Effects Models. R package version 3.1-117. Available at http://CRAN.R-project.org/ package $=$ nlme.

R Core Team (2014). R: A Language and Environment for Statistical Computing. Vienna, Austria: R Foundation for Statistical Computing. Available at http://www.R-project.org/.

Sannohe, S. (2002). Change in the postmortem formation of hypostasis in skin preparations 100 micrometers thick. American Journal of Forensic Medicine and Pathology 23, 349-354.

Sen, P. K., Singer, J. M. and Pedroso-de-Lima, A. C. (2009). From Small Sample to Asymptotic Methods in Statistics. Cambridge: Cambridge University Press.

Shiotani, S., Kohno, M., Ohashi, N., Yamazaki, K. and Itai, Y. (2002). Postmortem intravascular high-density fluid level (hypostasis): CT findings. Journal of Computer Assisted Tomography 26, 892-893. 
Singer, J. M., Rocha, F. M. M. and Nobre, J. S. (2017). Graphical tools for detecting departures from linear mixed model assumptions and some remedial measures. International Statistical Review 85, 290-324.

Thali, M. J., Yen, K., Schweitzer, W., Vock, P., Boesch, C., Ozdoba, C., Schroth, G., Ith, M., Sonnenschein, M., Doernhoefer, T., Scheurer, E., Plattner, T. and Dirnhofer, R. (2003). Virtopsy, a new imaging horizon in forensic pathology: Virtual autopsy by postmortem multislice computed tomography (MSCT) and magnetic resonance imaging (MRI): A feasibility study. Journal of Forensic Sciences 48, 386-403.

Vaida, F. and Blanchard, S. (2005). Conditional Akaike information for mixed-effects models. Biometrika 92, 351-370. MR2201364

Vonesh, E. F. and Chinchilli, V. M. (1996). Linear and Nonlinear Models for the Analysis of Repeated Measurements. New York: CRC Press.

\section{J. M. Singer}

C. D. S. André

Departamento de Estatística

Universidade de São Paulo

Brazil

E-mail: jmsinger@ime.usp.br

E-mail: tuca@ime.usp.br

\author{
F. M. M. Rocha \\ Escola Paulista de Política, \\ Economia e Negócios \\ Universidade Federal de São Paulo \\ Brazil \\ E-mail: francisco.marcelo.rocha@gmail.com
}

\author{
T. Zerbini \\ Departamento de Patologia \\ Universidade de São Paulo \\ Brazil \\ E-mail: tazerbini@yahoo.com.br
}

